\title{
The Future of High Angular Resolution Star and Planet Formation Science in the Optical/Infrared
}

\author{
Lynne A. Hillenbrand \\ California Institute of Technology, Pasadena, CA 91125
}

\begin{abstract}
.
This presentation summarizes how some of the most pressing questions in the field of star and planet formation can be addressed by high angular resolution optical/infrared capabilities, and how many of these capabilities will in fact be available with realization of the space and ground facilities currently being planned for the 2005-2020 time frame.
\end{abstract}

\section{Introduction}

The quest to understand the formation of stars and planets, especially exo-solar planetary systems showing some resemblance to our own Sun and solar system, is a fundamental goal of modern astrophysics. Star and planet formation studies make use of all observational techniques at all wavelengths ranging from X-ray to radio bands. This presentation focuses on optical/infrared questions and capabilities involving high spatial resolution techniques.

There are a number of problems in star and planet formation that would benefit substantially from improved spatial resolution over current capabilities in the optical and infrared. Here, I focus on three: the initial mass function, circumstellar disks and planet detection.

\subsection{The initial mass function}

The initial mass function (IMF), or spectrum of stellar/sub-stellar masses produced in the process of molecular cloud fragmentation is one of the most fundamental products of the star formation process. Rigorous observational effort over the last decade has provided insight into the form and detailed shape of the IMF at the point of "birth" of stellar clusters, all the way from the most massive stars through to sub-stellar mass objects. While the IMF rises with an approximately Salpeter slope from $>100 \mathrm{M}_{\odot}$ to $<2 \mathrm{M}_{\odot}$ there is now ample evidence for a change in slope between $0.5-2 \mathrm{M}_{\odot}$ and another more dramatic change below $0.5 M_{\odot}$. A function in which the slope is continually changing with mass, such as the log-normal form, probably best describes the data in hand. Outstanding IMF issues include the maximum and minimum masses formed by the star formation process; the variation (or lack thereof) in $\mathrm{dN} / \mathrm{dM}$ as a function of metallicity, temperature/pressure, or local stellar density; and the binary frequency and binary mass ratio $\left(\mathrm{q}=\mathrm{M}_{2} / \mathrm{M}_{1}\right)$ as a function of primary star mass and as a function of companion separation. Are (and if so, how are) companion mass ratio statistics related to the mass distribution of single stars? 
For studies of stellar populations in young clusters, the need for spatial resolution is dictated by crowding which limits one's ability first to identify cluster members and then to obtain photometry at the requisite level of accuracy $(<2-5 \%)$. These issues are particularly prescient in the sub-stellar mass regime where the objects of interest are 10-20 magnitudes fainter than the massive stars which cohabitate the cluster. Why might one choose to work in such difficult (due to point source crowding and high nebular backgrounds) regions? The reason is, simply, that the IMF statistics per unit angular area are far superior in regions like the Orion Nebula Cluster, NGC 3603, or R 136 than in less observationally complicated regions like Taurus, Ophiuchus, Lupus, or Chamaeleon.

For studies of fundamental binary properties, in particular determination of fundamental stellar masses, the need for spatial resolution is dictated by the semi-major axis regime one wants to probe. For $\mathrm{G}$ stars the peak in the stellar companion separation distribution occurs around $30 \mathrm{AU}$, within the bounds of our own planetary system, which is $\sim 0.2$ arc sec at the distance of the nearest star-forming regions. The current generation of large apertures $(8-10 \mathrm{~m})$ when used with adaptive optics enabling diffraction limited imaging can resolve equalmass systems with separation $>7-9$ AU. Of particular interest is the spatial resolution of known spectroscopic binaries for which combined radial velocity and astrometric orbital monitoring leads to fundamentally determined stellar masses. In addition to the interesting science of understanding the orbital (a, e) and mass ratio parameter space occupied at young ages, and hence the observations binary formation theories must reproduce, these systems are critical for the observational calibration of pre-main sequence evolutionary tracks which are often used "blindly" in IMF studies of star clusters.

\subsection{Circumstellar Disks}

The topic of disks and planet formation within disks has received a lot of attention at this meeting. The need for high angular resolution in this area is to obtain spatially resolved direct images of disks at various evolutionary stages. Measurement of disk size versus wavelength in combination with flux versus wavelength (the spectral energy distribution) is needed in order to correctly model these systems using absorption/re-emission and scattering codes. Some of the larger and brighter disk/envelope systems have been imaged in scattered light with various HST instruments (ACS, WFPC2, NICMOS) as well as with Keck and VLT. In particular we would like to image the innermost regions of accretion disks which serve both as the conduit linking material inflowing through the disk to the star, and as the launching point for the ubiquitous jets and outflows seen most prominently at the earliest evolutionary stages. Thus far several interferometric studies have made inroads into this heretofore elusive disk region, potentially resolving inner holes or rims/walls in disks. Imaging of the fine structure in disks, for example gaps, warps, and other features predicted to be a consequence of planet formation, is also high on the agenda. Finally, information on disk composition gradients requires spatially resolved dust and gas spectroscopy. We should be mindful of the time domain when thinking about high spatial resolution imaging; inner disk and outflow regions are known to be time-variable as might be expected given the short dynamical time scales involved on small geo- 
metric scales. Extended sources in which surface brightness variability has been observed include several disks and many jets. Temporally resolved and spatially resolved data on these regions are the likely path to understanding their physics.

\subsection{Planet detection}

The detection of exo-solar planets is one of the great triumphs of the latter part of the $20^{\text {th }}$ century. After nearly a decade of discovery, our questions in this area have become more refined and consequently more challenging in terms of the observations needed to address them. Future planet detection, characterization, and statistical analyses can be accomplished through several means including direct (coronographic) imaging, continuation of the successful radial velocity surveys, astrometric techniques, and transit photometry. I mention these techniques here since while several of them are already considered high angular resolution astronomy (coronographic imaging and high precision astrometry), others are in fact proxies for high angular resolution (high spectral dispersion a.k.a. high velocity resolution, and high photometric precision both of which when done as time series sample short dynamical time scales) since they are capable of sampling planetary system size scales on planetary system time scales. These techniques offer the potential to detect planets directly (high contrast imaging, in/out of transit spectroscopy) or indirectly (velocity wobble, astrometric wobble, inference of planet-induced gaps in disks).

\section{Paths to the Future at High Angular Resolution}

As we discuss the present observational capabilities and compare them with those available in the future, it is useful to recall that the angular and corresponding linear scales at the distance of the nearest star-forming regions (140 pc) are: $1 "=140 \mathrm{AU}, 0.1 "=14 \mathrm{AU}, 10 \mathrm{mas}=1.4 \mathrm{AU}$, and 1 mas $=0.14$ AU. All of these scales are of interest in studies of circumstellar disk physics and planet formation, and are useful in studies of the initial mass function and companion star statistics. The diffraction limit at $2 \mu \mathrm{m}$ of the current generation of premier optical/infrared ground-based facilities is $\sim 50$ mas which will improve to $\sim 16$ mas when the next generation of $30 \mathrm{~m}$ technology is realized. Interferometric capabilities are currently better than 5 mas fringe spacing with the smaller facilities and 3-10 mas with the higher sensitivity Keck and VLT Interferometers; visibility measurements enable inference of source sizes $<1$ mas. Of note is the difference in field of view between the single apertures (several to tens of arc minutes) and the interferometers $(<0.1-1$ arc seconds). For high dispersion spectroscopy as a proxy for high angular resolution, under the Keplerian assumption disk kinematics can be probed (with the right emission or absorption line) at a radius inversely proportional to velocity resolution squared. Even higher resolution, $\mathrm{R} \gtrsim 10^{5}$, is needed to discern finer structure in disks, such as gaps due to recent planet formation. The high precision photometric monitoring proxy for high angular resolution in principle offers infinitely small angular resolution.

So what does the future hold for the optical/infrared at high angular resolution? I have chosen to break up the discussion into several time-delimited segments, depending on the wait for the capability at hand to emerge for main 
stream science usage. My apologies to anyone whose mission or mission concept I do not mention. The number of relevant facilities is impressive but also daunting, and I can not possibly describe them all in the time/space allotted.

\subsection{Near-term Future Capabilities}

My near-term discussion encompasses everything from those facilities just coming on line to those happening within the decade, 5-7 years out.

Ground-Based Interferometers: KI, VLTI, and LBTI. Separate papers in these proceedings discuss the recently commissioned Keck Interferometer (KI) and Very Large Telescope Interferometer (VLTI). Fringes with the two $10 \mathrm{~m}$ Kecks ( $85 \mathrm{~m}$ baseline) on Mauna Kea were first obtained at $2 \mu \mathrm{m}$ in 2001 , March but operation of the full array including four $1.8 \mathrm{~m}$ outrigger telescopes is as yet uncertain. The interferometer will operate in $\mathrm{V}^{2}$ mode (standard fringe constrast) at $2 \mu \mathrm{m}$ and in nulling mode (utilizing phase inversion in one arm to effectively null the central star) at $10 \mu \mathrm{m}$. Full-array imaging resolution is expected to be $\sim 5$ mas with 10-30 $\mu$ as astrometric accuracy. The VLTI at Paranal saw first fringes with two Unit telescopes at $10 \mu \mathrm{m}$ in July 2002 and at $2 \mu \mathrm{m}$ in July 2003. It is scheduled to operate with all four $8 \mathrm{~m}$ Unit telescopes and 5 auxiliary telescopes (for a total of 28 baselines). Imaging size is 4 mas and astrometric accuracy $10 \mu$ as. The Large Binocular Telescope Interferometer (LBTI) is a mid-infrared nulling interferometer being built for operations in 2006 on Mt Graham. Important science goals of these interferometers include measurement of the sizes of the warm inner $(<0.1 \mathrm{AU})$ regions of circumstellar disks, study of exo-zodiacal ( $\sim 1 \mathrm{AU})$ dust via $10 \mu \mathrm{m}$ nulling, direct detection of brown dwarfs and Jupiter-mass planets on close orbits to young and old stars, indirect detection of Uranus mass planets, and study of pre-main sequence binary orbits for the purpose of determining fundamental stellar masses.

Space-Based Interferometers: SIM and Gaia. In addition to their groundbased pursuits, both the United States and the European nations are planning interferometric missions in space. ESA's Gaia consists of three $1.4 \mathrm{~m}$ telescopes, two of which are used for astrometry and one of which is for spectrophotometric data acquisition. Launch is scheduled for 2010 into an L2 orbit with an expected 5 year lifetime of all-sky scanning. Gaia's main goals are proper motions and parallaxes for $10^{9}$ stars with $4 \mu$ as precision that will connect us to the inertial reference frame. A planet search is a by-product of the Gaia program with main operations revealing any Jupiter-mass or larger planet/brown-dwarf/star companions having orbital periods between one-third and twice the mission lifetime. NASA's Space Interferometry Mission (SIM) consists of 3 collinear Michelson interferometers over a $10 \mathrm{~m}$ baseline operating at visible wavelengths. Astrometric accuracy will be $1 \mu$ as (narrow-angle) to $4 \mu$ as (wide-angle). Launch will be in 2009 into an Earth-trailing orbit with operations lasting 5-10 years. SIM is a pointed mission, surveying 2000 nearby stars, including about 150 young stars where orbital migration might be observed amongst the sample, for astrometric signatures of massive planetary companions (Jupiter's pull on the Sun is 500 $\mu$ as at $10 \mathrm{pc}$ ) and 250 nearby stars for terrestrial-mass planets (Earth's pull on the Sun is $0.3 \mu$ as at $10 \mathrm{pc}$ ). While radial velocity surveys can detect massive Jupiters, astrometry is required in order to find Earths. Additional general science of the space interferometers includes improved accuracy in stellar positions, 
distances, proper motions, measurement of star cluster dynamics, and study of Galactic disk and bulge kinematics.

Transit Missions: MOST, COROT, Kepler, and Eddington. Continuing the planet-finding theme, a number of space-based efforts are underway to find planets through high precision photometric monitoring. This search method is also being pursued from the ground by a large number of groups. As noted above, high precision photometric monitoring is a form of high spatial resolution given the small size scales probed. The photometric accuracy required to detect Jupiters is $1 \%$, to detect Uranus or Neptune analogs $0.1 \%$, and to detect Earths $0.01 \%$; these constraints limit the targets to 6-16'th magnitude stars. The space missions all operate at optical wavelengths and include CSA's MOST launched in 2003, France's COROT scheduled for 2005, NASA's Kepler in development for 2007, and ESA's Eddington scheduled for 2008. Mission goals for these spacecraft include transit detection (hundreds of thousands of stars searched) and asteroseismology (tens of thousands of stars searched) with studies of stellar rotation, stellar activity, and particular classes of binary stars important ancillary science. The capabilities of this suite of missions are complementary.

"Finder" Missions: SIRTF, SOFIA, Astro-F/IRIS, and WISE. While our focus here has been on high angular resolution, we should not forget to mention the suite of new low angular resolution capabilities that in part serve as the "finder" telescopes that will produce the targets to be pursued with the next generation of high angular resolution missions.

SIRTF (Space InfaRared Telescope Facility) was launched by NASA in 2003, as this manuscript was being written in fact. SIRTF is a $0.85 \mathrm{~m}$ telescope in a heliocentric Earth-trailing orbit which feeds three instruments covering 3-180 $\mu \mathrm{m}$ in eight photometric bands and the 5-40 $\mu \mathrm{m}$ range with $\mathrm{R}=100 / 600$ spectroscopy. SIRTF's mission will last 2.5-5 years. SOFIA (Stratospheric Observatory for Infrared Astronomy) is US-German venture consisting of a $2.7 \mathrm{~m}$ telescope mounted in an airborne 747SP flying at 39,000-45,000 feet. The telescope is optimized for mid- to far-infrared operations (diffraction-limited beyond 10-15 $\mu \mathrm{m})$ and the instrument suite covers the full spectral range from $0.3-1600 \mu \mathrm{m}$ at a variety of imaging bands and with varied spectral resolution from $R=1000$ to $>10,000$. First light is expected in 2005 with a $20-25$ year lifetime. SOFIA's sensitivity is intermediate between those of IRAS and SIRTF, but with spatial resolution a factor of 3-5 better than SIRTF. Goals common to both SIRTF and SOFIA include studies of star-forming regions, searches for young super-planets and brown dwarfs, detailed study of proto-planetary and debris disks, and assessment of the dust and gas disk frequency with age. Particularly exciting on these missions are the spectroscopic capabilities which will give us a detailed look at the mineralogy and rich chemistry in disks.

Japan's Astro-F or IRIS (InfraRed Imaging Surveyor) is a $0.7 \mathrm{~m}$ telescope in a polar orbit planned for around 2006. Over 2.5-5 years, with one instrument it will conduct a low-resolution, 30-50", all-sky survey at 50 and $200 \mu \mathrm{m}$ while with a second instrument it will obtain pointed observations at higher resolution, 2", through filters ranging from 2-26 $\mu \mathrm{m}$. WISE (Wide-field Infrared Survey Explorer) or NGSS as it was formerly known, is a proposed 4-band 3.5-23 $\mu \mathrm{m}$ all-sky survey at 5-10" resolution for launch by NASA in 2008 to a polar orbit. 
On a 6-12 month mission this $0.5 \mathrm{~m}$ telescope will reach all-sky sensitivities in the mid-infrared comparable to those of the optical SDSS.

SIRTF and SOFIA are pointed observatories while Astro-F and WISE are all-sky surveys. Collectively, these relatively low spatial resolution infrared missions will provide a wealth of targets for follow-up study with the next generation of high angular resolution facilities where, for example, newly found companions to Sun-like stars can be characterized and newly discovered disks can have their composition and structure as a function of disk radius understood.

\subsection{Mid-term Future Capabilities}

These are the beneficiaries of the aforementioned "finder" missions. I have only one space-based mission and some interesting prospects for the ground to discuss in this category of 8-15 years hence facilities.

The next generation space telescope (NGST) has been re-named JWST and is presently a $5.6 \mathrm{~m}$ segmented mirror telescope. JWST will be passively cooled at its L2 orbit, feeding three instruments that will obtain photometry and (multi-object) spectroscopy at resolution several hundred to several thousand. Covering 1-28 $\mu \mathrm{m}$ the near-infrared field of view is 5' and the mid-infrared 1.5'. Launch is presently scheduled for 2011 with a 5-10 year mission anticipated. The detectors will suffer backgrounds 6 orders of magnitude lower in the thermal infrared compared to ground-based facilities and will achieve nano-Jansky (1-3 $\mu \mathrm{m}$ near-infrared) to better than micro-Jansky (10-20 $\mu \mathrm{m})$ sensitivities. JWST's objectives are to detect the "first (near-infrared) light" from primordial stars and galaxies and to measure the creation of the first heavy elements. However, it will also have a strong program of general astrophysics related to star and planet formation including measurement of the stellar/sub-stellar mass function in extreme star-forming environments, determination of the minimum mass that can freely fragment from a molecular cloud, and studies of gas and dust disks around young stars. JWST is a NASA, ESA, and CSA partnership.

Now what about the ground? Our premier large-aperture ground-based facilities (Keck I and II at 10m, Subaru at 8m, Very Large Telescopes 1-4 and Gemini North and South all at 8m, and the forthcoming South African Large Telescope at $11 \mathrm{~m}$ ) are envisioned to lead to a next generation of telescopes that may be $15-20 \mathrm{~m}$ (e.g. the single aperture LAT and VLOT or the multiple aperture $20-20$ concepts) to $30 \mathrm{~m}$ (CELT or GSMT) or even larger $50-100 \mathrm{~m}$ (Euro50 or OWL) in size. Of great amusement is the comparison of one of these behemoths to a modern day sports stadium where the aperture and supporting structure takes up the majority of a rugby, soccer, or football field and the telescope enclosure is nearly the size of the entire stadium. We have quite a bit of technology development and ingenious engineering on the large telescope design and development agenda.

Speaking more seriously now about the comparative advantages of the ground and of space, it is important to note that there are complementary roles to be played by a $20-30 \mathrm{~m}$ ground-based facility and a $5-6 \mathrm{~m}$ space-based facility. For imaging, while the near-infrared (1-2.5 $\mu \mathrm{m})$ sensitivities of, as examples, a $30 \mathrm{~m}$ ground-based telescope and a $6 \mathrm{~m}$ space-based telescope are similar in the background-limited case, with diffraction-limited performance the $30 \mathrm{~m}$ has better spatial resolution by the ratio of the aperture sizes, or approximately a 
factor of 5. Since we will always build bigger light buckets on the ground than we send into space, this advantage should be maintained. In the mid-infrared $(>3 \mu \mathrm{m})$ ground-based sensitivity is not competitive with that available from space. However, as mentioned above, the ground-based spatial resolution will always be better in the diffraction-limited case. Further, other capabilities such as high dispersion mid-infrared spectroscopy may become available only from the ground. For near-infrared spectroscopy, at moderate spectral resolution $\mathrm{R} \approx 4000$, the "air glow" due mainly to $\mathrm{OH}$ emission lines is suppressed in the red optical and near-infrared which means that traditional backgrounds are reduced (by a factor of $\sim 30$ ) making the ground competitive with space. Further, truly high dispersion spectrographs or other specialized capabilities such as integral field units are unlikely to be built as space instruments, defining yet other niches for the ground. These trades between wavelength and spectral resolution have been quantified by the CELT and GSMT projects. Great complementarity is expected between the next generation of $30 \mathrm{~m}$ ground and 5-6 $\mathrm{m}$ space facilities from 1-25 $\mu \mathrm{m}$.

\subsection{Far-term Future Capabilities}

It is never too early to plan for the future according to some financial advisors, and the same may be said for big-ticket space facilities paid for with public money. In the era of longterm strategic planning that is presently fashionable in science, there is always an identified horizon filled with the necessary as well as the exotica. Mission concepts which have emerged thus far in the $>15-20$ year time frame are a combination of both.

NASA's Terrestrial Planet Finder (TPF) and ESA's Darwin mission both have as their primary goal the discovery and characterization of Earth-like, potentially habitable planets. TPF is being studied as either an infrared $(7-20 \mu \mathrm{m})$ separated spacecraft interferometer achieving nulling to one part in $10^{6}$ and detecting planets via their thermal emission, or an optical wavelength coronograph expecting starlight suppression to one part in $10^{9}$ and detecting planets via reflected light from the central star. TPF is aiming for 2015 launch into either L2 or an Earth-trailing orbit. Darwin is cast as a six-element infrared interferometer plus central beam combiner planned for 2015 or later and also located at L2. The potential for collaboration between these missions is to be encouraged, given their great expense and vast scientific overlap. Achieving the first of their goals means that a statistically significant number of stars must be surveyed and the observations must be capable of discovering Earths located in the "habitable zone" of liquid water existence. The second goal, characterization of Earth-like planets requires a search for atmospheres, habitability, and perhaps even biosignatures through indicators such as $\mathrm{CO}_{2}, \mathrm{H}_{2} \mathrm{O}, \mathrm{N}_{2} \mathrm{O}, \mathrm{O}_{2}, \mathrm{O}_{3}$, and $\mathrm{CH}_{4}$; these molecules in particular were all seen by the Mars Express Mission as it looked back and took a spectrum of Earth just before this meeting (July 2003). For more general astrophysics purposes, it should be appreciated that these missions will have spatial resolution 10-100 times that of JWST and are thus capable of far more star and planet formation science than just their articulated primary goals.

At short wavelengths, the Space UltraViolet Optical (SUVO) telescope is a conceived successor to Hubble (HST) operating in the ultraviolet and visible 
range. While focusing on the evolution of the first galaxies, quasars, and stars, this observatory would also provide substantial high spatial resolution $(<30$ mas) capability for star and planet formation studies over the needed wide fields $\left(>10^{\prime}\right)$. A 4-8m (deployable optics) aperture is envisioned with launch hoped for by the end of next decade.

At long wavelengths, the Single Aperture Far-InfraRed (SAFIR) observatory and the Submillimeter Probe of Early Cosmological Structure (SPECS) offer our first chance for truly high spatial resolution in the far infrared. SAFIR is a follow-on to NGST, SIRTF/Herschel, and SOFIA, an 8-10 m telescope operating from $\sim 20-500+\mu \mathrm{m}$ (diffraction-limited beyond $40 \mu \mathrm{m}$ ) with both imaging and $\mathrm{R} \approx 1000$ spectroscopy. Both filled and sparse-aperture mirrors are being explored. The facility is expected to be limited only by astrophysical backgrounds (zodiacal light, galactic cirrus, and the cosmic microwave background) and thus will achireve heretofore unrealized sensitivity in the mid- and far-infrared. Launch is contemplated for the 2015-2020 time frame to L2. SPECS intends to operate several $1 \mathrm{~km}$ baselines and achieve HST-like resolution (50 mas) in the far-infrared, a truly remarkable capability, if realized. Science goals of SAFIR and SPECS include study of the interstellar medium in star-forming regions, protostellar objects, and the more evolved proto-planetary and debris disks.

\subsection{Dreamland}

There are a few concepts even further afield than the somewhat mature but still developing and evolving mission concepts outlined above. Planet Imager and Life Finder are two in the NASA long term plan, with launch dates approaching 2050. Planet Imager hopes to obtain spatially resolved images of planets orbiting other stars while Life Finder is a spectroscopy mission geared towards finding signatures of life (biomarkers).

\section{Concluding Remarks}

As reviewed in this talk, there are a variety of high angular resolution techniques or proxies thereof under development, or just coming online now, that are of interest for star and planet formation studies. Direct imaging with large apertures, interferometry, high dispersion spectroscopy, and transit photometry are among the maturing technologies. A series of ground-based telescope concepts and space mission concepts are waiting in line over the next several decades for their turn to implement these technologies and techniques. How do we decide as a community what capabilities to pursue? In order to secure either public or private funding at the scale needed to carry out our current plans and dreams there must exist an alignment of the following four concepts:

- important science questions

- technological readiness

- cost

- correspondence with strategic goals 
We astronomers are fortunate to live in a time when this is not only possible, but desired and expected by the science-consuming public at large. Complementary science will be done from the ground and from space with the next generation facilities. Another general conclusion from the present survey of the future is that L2 has the potential to become quite crowded by about 2020 !

Acknowledgments. I am grateful to the following for providing some of the slide material used in this talk: Mike Devirian, Mark McCaughrean, Richard Ellis, Alycia Weinberger and of course the WWW at large. I also wish to commend Hans Zinnecker for his statement during the conference summary talk about a talk not being a paper; in this instance even a conference talk proceedings is not a comprehensive paper on the topic at hand! Finally, my gratitude to the conference organizers for the invitation to experience $\mathrm{Oz}$. 